\title{
Disease Related Protein Folding and Quality Control Protein Biosynthesis in the Endoplasmic Reticulum
}

\author{
Nanda Rachmad Putra Gofur ${ }^{1}$, Aisyah Rachmadani Putri Gofur ${ }^{2}$, Soesilaningtyas ${ }^{3}$, \\ Rizki Nur Rachman Putra Gofur ${ }^{4}$, Mega Kahdina ${ }^{4}$, Hernalia Martadila Putri ${ }^{4}$ \\ ${ }^{I}$ Department of Health, Faculty of Vocational Studies, Universitas Airlangga, Surabaya, Indonesia \\ ${ }^{2}$ Faculty of Dental Medicine, Universitas Airlangga, Surabaya, Indonesia \\ ${ }^{3}$ Department of Dental Nursing, Poltekkes Kemenkes, Surabaya, Indonesia \\ ${ }^{4}$ Faculty Of Medicine, Universitas Airlangga, Surabaya, Indonesia \\ *Corresponding Author: Nanda Rachmad Putra Gofur, Department of Health, Faculty of Vocational \\ Studies, Universitas Airlangga, Surabaya, Indonesia.
}

\begin{abstract}
Introduction: The endoplasmic reticulum is an organelle that functions as a place for protein folding. The process of folding protein involves several components including: chaperones, calcium, and several related enzymes. Capheron molecules function to help the protein folding process, slow down the folding speed of proteins so as to prevent protein aggregation. The supervisor also has a role to maintain calcium homeostasis in the ER, where homeostasis can be achieved in the RE lumen calcium concentration of about $400 \mu M$ due to the role of the companion interaction with the SERCA receptor and IP3 receptor. Calcium itself plays a direct role in the protein folding process by modulating chaperon bonds with carbohydrates in $\mathrm{N}$-glycoproteins so that chaperons can function to help protein folding.

Discussion: Quality Control is also carried out by calnexin and calreticulin, which play a role in the correct folding of proteins and also recognize protein misfolding which then tries to fold it back. This role is supported by the presence of calcium in the RE lumen. The misfolding protein will increase calreticulin expression, then calreticulin through a certain mechanism influences the SERCA receptor so that there is an increase in the concentration of calcium in the RE lumen. Calcium then helps calreticulin in carrying out its function by modulating the interaction between calreticulin and carbohydrates so that refolding can occur. Proteins that have been completely molded are then removed from the ER, on the other hand, if they cannot be refolded, they will enter the ERAD mechanism and then be brought to the proteosome and degraded.

Conclusion: Proteins are subjected to quality control checks and any found to be misformed or folded incorrectly will be rejected. This rejection hail is stored in the lumen or sent for recycling for its eventual breakdown into amino acids. There are many disease related to protein foldings and quality control in RE. A type of emphysema (lung problem) is caused by the RE quality control department constantly rejecting the protein incorrectly folded. The protein needed was never exported from the crude RE. Quality control plays a role in cystic fibrosis. A form of cystic fibrosis is caused by a single missing amino acid, phenylanaline, in a specific position in the protein construction. Proteins can work well without amino acids but these services must be precisely provided by quality control for speck of error and resist storage of protein in the crude RE lumen.
\end{abstract}

Keywords: Protein folding, quality control, reticulum endoplasmic, disease

\section{INTRODUCTION}

The endoplasmic reticulum is an organelle that functions as a place for protein folding. The process of folding protein involves several components including: chaperones, calcium, and several related enzymes. Capheron molecules function to help the protein folding process, slow down the folding speed of proteins so as to prevent protein aggregation ${ }^{1}$.

The supervisor also has a role to maintain calcium homeostasis in the ER, where homeostasis can be achieved in the RE lumen calcium concentration of about $400 \mu \mathrm{M}$ due to the role of the companion interaction with the SERCA receptor and IP3 receptor. Calcium itself plays a direct role in the protein folding process by modulating chaperon bonds with carbohydrates in N-glycoproteins so that chaperons can function to help protein folding ${ }^{2}$.

The protein folding process also involves several enzymes, for example glucosidase, which acts to release glucose residues which serve as markers for companions to hold the 
protein. The folding protein will end glucose in the $\mathrm{N}$-glycoprotein, all of which have been removed by glycosidase. The GT enzyme (glucose UDP glycoprotein glucosyltransferase) also plays a role in the folding process, where too fast removal of glucose by glucosidase can cause fold or opening errors so that the GT enzyme will add new glucose and thus refolding can $^{3}$.

Proteins that pass through the secretion pathway generally contain disulfide bonds which play an important role in the folding process and protein function. In eukaryotes, the endoplasmic reticulum (ER) is the entry point to the secretion pathway and is a cellular compartment where folding and ordering of disulfide bonds occurs. Disulfide bonds can form spontaneously in vitro in the presence of an oxidizing compound such as an oxygen molecule or glutation in its oxidized form. However this process is generally slow and inefficient. In vivo, the ordering of disulfide bonds, depending on the cell machine to catalyze, and new disulfide bonds (oxidation) and non-native disulfide bonds (isomerization). The oxidation and isomerization required for ordering the negative disulfide bond. The formation of a negative disulfide bond is important for the folding of many proteins ${ }^{1}$.

The disulfide bond provides additional extracellular stability of the protein by crosscovalent cross-linking of two cysteine residues. Disulfide formation is sometimes error prone, especially in the early stages of folding, and correct cysteine attachment to the disulfide bond requires that any mispaired disulfide must be broken down and reconstituted with different rules to build the negative structure. In bacteria, disulfides are formed in the periplasm through the elaboration of an oxidation and isomeration system which ensures that the two cysteine residues are properly connected. The electron transport pathway is connected, disulfide bonds with the respiration chain. The membrane protein, DsbB oxidizes the CxxC active side of the DsbA protein (homologous PDI) which then catalyzes the order of disulfide bonds in the protein that will experience folding. DsbB is then re-oxidized by ubiquinone produced during respiration ${ }^{3}$.

The group carbohydrates in this $\mathrm{N}$-glycoprotein can be measured with a companion (calreticulin) so that the protein folding process can begin. Calnexin, which is a transmembrane protein, will also experience this complexity and will also help the protein folding process. Glucosidase I and II enzymes will cut the first and second sugar residues from the oligosaccharide group, then glucosidase II will also cut the third (last) glucose residue previously carried out by QC which will dismantle the protein properly. Glucose in this case is used as a marker for calnexin where the $\mathrm{N}$-glycoprotein has lost all its glucose residues, so there is no calnexin complex and the folding protein is declared complete. The protein that has been completely folded is removed from the ER. In some cases, the third glucose residue in the oligosaccharide is removed faster causing the formation of a misfolding or unfolding protein and this condition will be recognized by another QC component, namely the GT enzyme (UDP-glucose glycol-protein transferase). The GT enzyme will replace the remaining glucose wasted with a new glucose group so that the misfolding or unfolding protein can be folded again. The QC cycle can occur repeatedly until the protein folds completely ${ }^{2}$.

In eukaryotes, these post-translational modifications occur in the endoplasmic reticulum (ER) where there are a number of enzymes that catalyze the proper order of disulfide bonds. In yeast and mammalian cells, the oxidation equivalents for the bond orders are same. In principle, lfida is carried out by 8 Ero1p (endoplasmic reticulum oxidoreductin 1 protein). This disulfide is then fed to protein disulfide isomerase (PDI1), which is an important catalyst folding in RE. Erolp is a protein that is bound to the RE lumen membrane with a molecular weight of $65 \mathrm{kDa}$ which plays an important role in the stability of S. cerevisiae ${ }^{1}$.

In vivo, Ero1p oxidizes disulfide-containing proteins. Ero1p was first identified using genetic screening where, if overproduced, this protein can change the nature of resistance to DTT (reductant molecules) or if mutated can cause sensitivity to DTT. The addition of the thiol oxidant, the diamide, to the growth medium can complement Ero1p deficiency, probably because the diamide performs an oxidative function of the missing gene product. These results indicate that the main function of Erolp is to oxidize newly synthesized proteins ${ }^{2}$. This article aims to review protein folding and quality control of protein biosynthesis in the endoplasmic reticulum.

\section{DISCUSSION}

In general, it can be explained that new polypeptides enter the UG through translocation 
of the Sec61 complex, which contains 20 components (some of which are shown). Subsequently, the core oligosaccharide, GlcNAC2Man9Glc3, was cotranslationally transferred from the dolichol lipid precursor (orange oval) to the NXS / T (X 61/4 P) consensus site by OST. Terminal glucoses (green triangles) are cut by glucosidase I and II (Glc I and Glc II) into the structure of
GlcNAC2Man9Glc1 which binds to the membrane protein, calnexin $(\mathrm{CNX})^{1}$.

The non-glycosylated substrate binds to $\mathrm{BiP}$ and undergoes non-lectin-mediated folding, and / or is replaced posttranslationally by the OST (B) isoform and thus enters the CNX cycle. If translocation does not occur, the substrate is sent to cytosolic material (eg Hsp70) and may be degraded ${ }^{3}$.

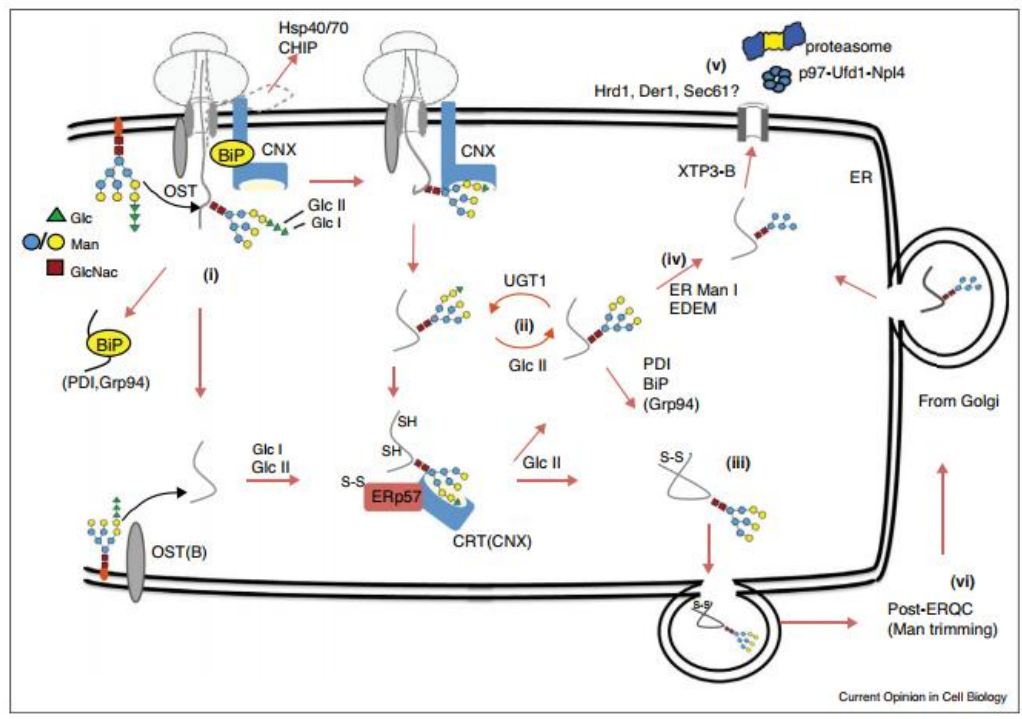

Figure1. Protein Folding Mechanism ${ }^{3}$

After being released from CNX, the N-linked glycol is further deolosylated by GlcII, and the substrate is spontaneously folded, transferred to the contents containing BiP, the PDI complex and / or the companion complex Grp94, or recognized as incorrect by UGT1 and reglucosylated. Reglucosylation makes it possiblerebinding to $\mathrm{CNX}$ and lumenal homologous calreticulin (CRT), which stimulates isomerization and disulfide formation via the associated ERp57 PDP. Homologous. When folded, the substrate is packed into COPII coated vesicles to be sent to Golgi ${ }^{2}$.

During this process, the mannose residue (yellow circle) can be subjected to removal by ER Mannosidase I (ManI) and some homologous EDEMs. Mannose pruning decreased affinity for UGT1 and ultimately resulted in the GlcNAC2Man5 structure binding additional lectins, including XTP3-B and OS-9 (not described), which brought the substrate to the retrotranslocation machine for retro translocation to the cytosol, ubiquitination by the E3 ligament, and degradation. by proteasome 26s. In the event of ER stress or protein overload, misfolded substrates containing GlcNAC2Man9 glycans can also be sent to the Golgi apparatus and sorted for lysosome degradation, or modified by Golgi ManII and returned to the ER for degradation via ERAD ${ }^{1,2}$.

\section{Signal Nuclear Local Control}

When a nucleus is disassembled during mitosis, the lamina depolymerizes the nucleus. This disassembly is at least partly a result of direct phosphorylation of the nuclear lamina by cyclic dependent kinases activated at the onset of mitosis. At the same time, the inner nuclear membrane proteins are phosphorylated, and the nuclear pore complex breaks down and spreads out in the cytosol. Nucleic envelope membrane proteins - no longer tethered to pore, lamina, or chromatin complexes - spread across the ER membrane. Together, these events break down the barrier that normally separates the nucleus and cytosol, and these nuclear proteins that are not bound to the membrane or chromosomes attach completely to the cytosol of the separating ${ }^{4}$.

Then in mitosis (at the end of anaphase), the nuclear is reassembled on the surface of the chromosome, as the phosphorylated inner nuclear membrane proteins and lamin are broken down into chromatin. The ER membrane wraps around the chromosome groups and 
continues to fuse until the nuclear is closed and reformed. During this process, the nuclear pore complex also reassembles and begins to actively reabsorb the protein containing the nuclear localization signal. Because the nuclear is initially closely related to the surface of the chromosomes, the newly formed nucleus does not include all proteins except those that are initially bound to the mitotic chromosome and which are selectively imported through the complex of nuclear pores. In this way, all other large proteins are kept away from the newly assembled core ${ }^{5}$.

The nuclear localization signal is not removed after being transported to the nucleus. This is because nuclear proteins need to be imported repeatedly, once after each cell division.
Conversely, once a protein molecule has been imported into another membrane-covered organelle, it is passed down from generation to generation within that compartment and does not need to be translocated again; The signal sequences in these molecules are often deleted after protein translocation ${ }^{4,6}$.

\section{Translocation Proteins in Mitochondria}

Mitochondrial organelles also undergo the same process for transporting proteins such as the endoplasmic reticulum. Proteins pass through the mitochondrial membrane in an unfolded form whose structure is stabilized by a signal protein called a chaperon. This protein facilitates the polypeptide chain leading into the mitochondria ${ }^{\text {? }}$.

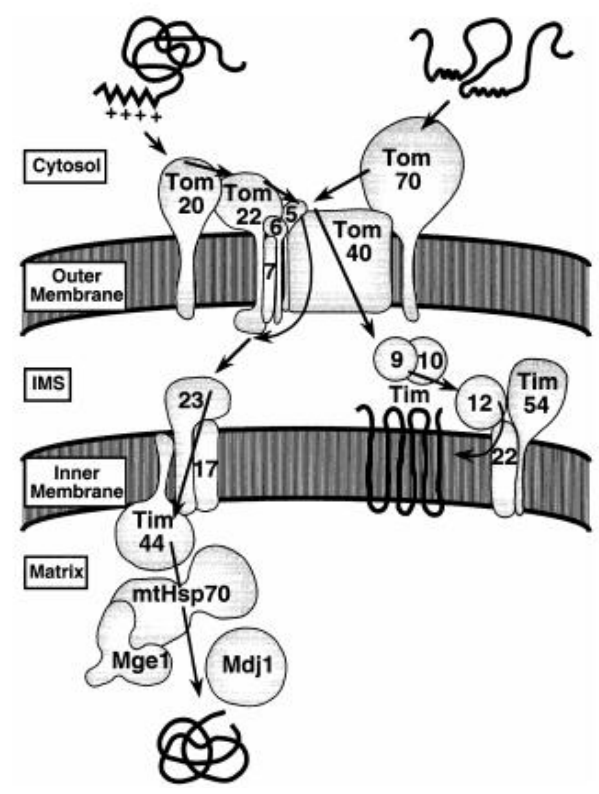

Figure2. The mechanism of action of protein translocation to the mitochondria ${ }^{7}$.

Many chaperon proteins are identified as heatshock protein (Hsp) because they are able to increase temperature or change shape when there is a change in their environment and are able to bind to proteins that have not been folded. In certain types such as the family of Hsp60 will form like a "double" which is composed of 14 protein subunits called chaperonins ${ }^{7}$.

Meanwhile, the unfolded polypeptide that is transferred to the mitochondria also has a signal called the matrix-targeting sequence (MTS) or presequence, which is characterized by an $\mathrm{N}$ terminal amphipathic helix (N-Met-Leu- ArgTre-Ser-Ser-Leu. - Phe-Tre- Arg- Arg- ValGlut- Pro- Ser- Leu-Phe- Arg- Asp- Iso- LeuArg- Leu- Glut- Ser- Treo). The MTS is used to recognize two receptors, namely translocase-ofthe-outer membrane (TOM) and translocase-of- the-inner membrane (TIM) which are on the outer and inner membranes of the mitochondria ${ }^{8}$.

The stages in the figure begin with the synthesis of polypeptides by ribosomes in the cytosol which already contain MTS and interacts with chaperon protein (Hsp70). Furthermore, MTS interacts with the TOM 20/22 receptor on the outer membrane (OMM / mitocondrial outer membrane) and is then transferred to the receptor next to it, namely TOM 40 . Then the polypeptides are translocated into the space between membranes through the TOM 40 channel and interact with the TIM 23 / receptor. 17 which is in the inner membrane (IMM / inner membrane mitocondria). Meanwhile, Hsp70 chaperon protein interacts with TIM44. Then the hydrolysis of ATP by Hsp70 will help translocation of polypeptides to the 
mitochondrial matrix. MTS or the targetting sequence in the polypeptide will be broken by the protease matrix enzyme ${ }^{8,9}$.

In the presence of a complex dual membrane system, the mitochondria use different and complex mechanisms for the importation of cytosolic precursor proteins, ranging from electrostatic interactions to generations that have mechanical strength. Perhaps most of the participating protein components have been identified in the yeast $S$. cerevisiae. The section consists of three large membrane embedded protein complexes and a large set of dissolved proteins from the matrix and intermembrane spaces. In this discussion, the outer membrane translocase pore together with the preprotein receptor molecule, the two membranes in the translocation complex, the Tim23 and Tim17 complexes cooperate with the Hsp70 system in the matrix and the Tim22 and Tim54 complexes cooperate with proteins from the intermembrane space ${ }^{7}$.

\section{Quality Control in Reticulum Endoplasma}

Quality Control (QC) in RE is basically done by the components involved in the protein folding process. The protein synthesized in the ribosome enters the RE lumen. On the other hand, the delichol found in the RE membrane will capture the glucose in the cytosol to form oligosaccharides and by the oligosaccharide flipase enzyme will be reversed facing the RE lumen. Carbohydrates (oligosaccharides) are added to these newly synthesized proteins to form $\mathrm{N}$-glycoside bonds for the role of the enzyme oligosacharyltransferase (OST) to form $\mathrm{N}$-glycopoteins. The addition of these groups is to maintain stability, solubility, and protein load and protect it from denaturation and proteolysis. This cluster also plays a role in the correct protein folding process, efficient quality control, introduction to ERAD, etc. The oligosaccharide group consists of glucose, mannose, and $\mathrm{N}$ acetylglucosamine ${ }^{10}$.

Quality Control is also carried out by calnexin and calreticulin, which play a role in the correct folding of proteins and also recognize protein misfolding which then tries to fold it back. This role is supported by the presence of calcium in the RE lumen. The misfolding protein will increase calreticulin expression, then calreticulin through a certain mechanism influences the SERCA receptor so that there is an increase in the concentration of calcium in the RE lumen. Calcium then helps calreticulin in carrying out its function by modulating the interaction between calreticulin and carbohydrates so that refolding can occur. Proteins that have been completely molded are then removed from the $\mathrm{ER}$, on the other hand, if they cannot be refolded, they will enter the ERAD mechanism and then be brought to the proteosome and degraded $^{1}$.

It is also in the lumens that the quality control inspection process is carried out. Proteins are subjected to quality control checks and any found to be misformed or folded incorrectly will be rejected. This rejection hail is stored in the lumen or sent for recycling for its eventual breakdown into amino acids. A type of emphysema (lung problem) is caused by the RE quality control department constantly rejecting the protein incorrectly folded. The protein needed was never exported from the crude RE lumen ${ }^{10}$.

Quality control plays a role in cystic fibrosis. A form of cystic fibrosis is caused by a single missing amino acid, phenylanaline, in a specific position in the protein construction. Proteins can work well without amino acids but these services must be precisely provided by quality control for speck of error and resist storage of protein in the crude RE lumen ${ }^{2}$.

Recombinant proteins in the ER of mammalian cells are mainly focused on two RE components, namely: protein disulfide isomerase (PDI), an enzyme that catalyzes the formation of negative disulfide bonds in secretory proteins; and BiP / G1LP78, which is a homolog of Hsp70, which acts as a molecular chaperone for protein secretion through the ER. The formation of disulfide bonds is a covalent modification and is a post-translational modification experienced by proteins entering the secretory pathway. The formation of native disulfide bonds is an integral aspect of the protein folding pathway, and plays an important role in protein assembly, where most secreted proteins (for example, antibodies, procollagens) are oligomers of two or more polypeptide chains joined together by disulfide bond chains. This chain will control the proteins in the cells ${ }^{1}$.

Nuclear laminae are part of interconnected protein subunits called nuclear lamins. These laminae are a special class of intermediate filament proteins which are polymerized into two-dimensional portions. The nuclear lamina gives shape and stability to the nuclear envelope, which is attached to adhesions to the nuclear pore complexes and the integral membrane proteins of the inner nucleus. Lamina 
also interacts directly with chromatin, which interacts with membrane integral proteins of the inner nucleus. Together with lamina, these membrane proteins provide a structural link between DNA and the nuclear envelope ${ }^{5,11}$

\section{CONCLUSION}

Proteins are subjected to quality control checks and any found to be misformed or folded incorrectly will be rejected. This rejection hail is stored in the lumen or sent for recycling for its eventual breakdown into amino acids. There are many disease related to protein foldings and quality control in RE.

A type of emphysema (lung problem) is caused by the RE quality control department constantly rejecting the protein incorrectly folded. The protein needed was never exported from the crude RE. Quality control plays a role in cystic fibrosis. A form of cystic fibrosis is caused by a single missing amino acid, phenylanaline, in a specific position in the protein construction. Proteins can work well without amino acids but these services must be precisely provided by quality control for speck of error and resist storage of protein in the crude RE lumen.

\section{REFERENCES}

[1] Kleizen, B. and Braakman, I. (2004) 'Protein folding and quality control in the endoplasmic reticulum', Current Opinion in Cell Biology, 16(4), pp. 343-349. doi: 10.1016/j. ceb.2004 .06 .012 .

[2] Brodsky, J. L. and Skach, W. R. (2011) 'Protein folding and quality control in the endoplasmic reticulum: Recent lessons from yeast and mammalian cell systems', Current Opinion in Cell Biology. Elsevier Ltd, 23(4), pp. 464-475. doi: 10.1016/j.ceb.2011.05.004
[3] Araki, K. and Nagata, K. (2012) 'SUP: Protein folding and quality control in the ER.', Cold Spring Harbor perspectives in biology, 4(8), $\mathrm{p}$. a015438. doi: 10.1101/cshperspect.a015438.

[4] Cokol, M., Nair, R. and Rost, B. (2000) 'Finding nuclear localization signals.', EMBO reports, 1(5), pp. 411-5. doi: 10.1093/emboreports/kvd092.

[5] Alberts B, Johnson A, Lewis J, et al. Molecular Biology of the Cell. 4th edition. New York: Garland Science; 2002.

[6] Luttrell DK dan Luttrell LM. 2004. Not so strange bedfellows: G-protein-coupled receptors and Src family kinases. J Oncogene 23, 7969 7978. Nature Publishing :0950-9232 /04

[7] Bauer, M. F. et al. (2000) 'Protein translocation into mitochondria: The role of TIM complexes', Trends in Cell Biology, 10(1), pp. 25-31. doi: 10.1016/S0962-8924(99)01684-0.

[8] Voos, W. et al. (1999) 'Mechanisms of protein translocation into mitochondria.', Biochimica et biophysicaacta, 1422(3), pp. 235-54. doi: S0304-4157(99)00007-6 [pii].

[9] Gerits N, SergiyKostenko, Alexey Shiryaev, Mona Johannessen, Ugo Moens. 2008. Relations between the mitogen-activated protein kinase and the cAMP-dependent protein kinase pathways: Comradeship and hostility. Cellular signalings : 1592-1607

[10] Brodsky, J. L. and Skach, W. R. (2012) 'Protein Folding and Quality Control in the Endoplasmic Reticulum: Recent Lessons from Yeast and Mammalian Cell Systems', Current opinion in cell biology, 23(4), pp. 464-475. doi: 10.1016/j.ceb.2011.05.004.

[11] Natarajan, K danBerk, BC. 2006. Crosstalk Coregulation Mechanisms of G Protein Coupled Receptors and Receptor Tyrosine Kinases. Methods Mol Biol. 2006;332:5177.PMID:16878685 DOI: 10.1385/1-59745048-0:51

Citation: Nanda Rachmad Putra Gofur et al., "Disease Related Protein Folding and Quality Control Protein Biosynthesis in the Endoplasmic Reticulum", International Journal of Research Studies in Medical and Health Sciences. 2021; 6(2): 15-20. DOI: https://doi.org/10.22259/ijrsmhs.0602004

Copyright: () 2021 Nanda Rachmad Putra Gofur et al., This is an open-access article distributed under the terms of the Creative Commons Attribution License, which permits unrestricted use, distribution, and reproduction in any medium, provided the original author and source are credited. 